\title{
Hadron Decay Processes and the Quark Model.
}

\author{
R. VAN ROYEN \\ CERN - Geneva \\ V. F. WeISSKOPF \\ Laboratory for Nuclear Science and Physics Department \\ Massachusetts Institute of Technology - Cambridge, Mass. \\ (Nuovo Cimento, 50 A, 617 (1967))
}

Formula (8) on p. 625 should read

$$
f_{M}=\frac{G_{A}^{\prime}}{G_{Y}^{\prime}} 2 \frac{\psi_{M}(0)}{m_{M}^{\frac{1}{y}}}=1.4 \frac{\psi_{M H}(0)}{m_{M}^{\frac{1}{2}}} .
$$

The expressions for $a_{1 v}$ and $a_{2 v}$ on the bottom of p. 630 should read

$$
\begin{aligned}
& a_{1 v}=\frac{1}{k_{1}^{2}+m_{V}^{2}} \varepsilon_{\alpha \beta \gamma^{\prime} \mu^{\prime}} \varepsilon_{\alpha^{\prime} \bar{\rho}^{\prime} \gamma^{\prime} \mu} q_{\alpha}^{(1)} \varepsilon_{\beta}^{(1)} k_{1 \gamma} q_{\alpha^{\prime}}^{(2)} \varepsilon_{\beta^{\prime}}^{(2)} k_{1 \gamma^{\prime}}, \\
& a_{2 V}=\frac{1}{k_{2}^{1}+m_{V}^{2}} \varepsilon_{\alpha \beta \gamma^{\prime} \mu} \varepsilon_{\alpha^{\prime} \beta^{\prime} \gamma^{\prime} \mu} q_{\alpha}^{(2)} \varepsilon_{\beta}^{(2)} k_{2 \gamma} q_{\alpha^{\prime}}^{(1)} \varepsilon_{\beta^{\prime}}^{(1)} k_{2 \gamma^{\prime}} .
\end{aligned}
$$

Figure 2 on p. 632 is upside-down.

The partial widths for the $\eta$ quoted at the bottom of p. 638 do not include any $n-n^{\prime}$ mixing, except for $\Gamma_{n \rightarrow 2 \gamma}$.

With a mixing angle of $-10^{\circ}$ (see p. 634), we get then

and

$$
\Gamma_{n_{1} \rightarrow 2 \pi_{+}+\gamma}=1.6 \cdot 10^{-4} \mathrm{MeV} \text { without mixing }
$$

$$
\Gamma_{\eta \rightarrow 2 \pi+\gamma}=2.0 \cdot 10^{-1} \mathrm{MeV} \text { with mixing }
$$

The values for branching ratios including mixing become?

$$
\Gamma_{n \rightarrow 2 \gamma}: \Gamma_{n \rightarrow \pi+2 \gamma}: \Gamma_{n \rightarrow 2 \pi+\gamma}=1: 0.7 \cdot 10^{-3}: 0.19 \text {. }
$$

We regret that the $v$-spin operator was represented by the symbol $\sigma$, instead of $v$ in formulas (1), (2) and (3) on p.622 and 623. The $\sigma$ symbol used here is hard to distinguish from the bold-faced $\sigma$ which represents the ordinary spin. 